\title{
A Macroevolutionary Perspective on Multiple Sexual Traits in the Phasianidae (Galliformes)
}

\author{
Rebecca T. Kimball, Colette M. St. Mary, and Edward L. Braun \\ Department of Biology, University of Florida, P.O. Box 118525, Gainesville, FL 32611, USA \\ Correspondence should be addressed to Rebecca T. Kimball, rkimball@zoo.ufl.edu
}

Received 2 October 2010; Accepted 26 February 2011

Academic Editor: Rob Kulathinal

Copyright (C) 2011 Rebecca T. Kimball et al. This is an open access article distributed under the Creative Commons Attribution License, which permits unrestricted use, distribution, and reproduction in any medium, provided the original work is properly cited.

\begin{abstract}
Traits involved in sexual signaling are ubiquitous among animals. Although a single trait appears sufficient to convey information, many sexually dimorphic species exhibit multiple sexual signals, which may be costly to signalers and receivers. Given that one signal may be enough, there are many microevolutionary hypotheses to explain the evolution of multiple signals. Here we extend these hypotheses to a macroevolutionary scale and compare those predictions to the patterns of gains and losses of sexual dimorphism in pheasants and partridges. Among nine dimorphic characters, including six intersexual signals and three indicators of competitive ability, all exhibited both gains and losses of dimorphism within the group. Although theories of intersexual selection emphasize gain and elaboration, those six characters exhibited greater rates of loss than gain; in contrast, the competitive traits showed a slight bias towards gains. The available models, when examined in a macroevolutionary framework, did not yield unique predictions, making it difficult to distinguish among them. Even with this limitation, when the predictions of these alternative models were compared with the heterogeneous patterns of evolution of dimorphism in phasianids, it is clear that many different selective processes have been involved in the evolution of sexual signals in this group.
\end{abstract}

\section{Introduction}

The evolution of sexually dimorphic traits, such as armaments, bright coloration, or exaggerated traits has long been of interest to evolutionary biologists, as such traits are often thought to reduce fitness through natural selection (e.g., [1]). However, in spite of the potential negative consequences of these traits, many taxa exhibit not just one, but often multiple, sexually dimorphic traits. In nearly all systems examined, there is evidence that these multiple, sexually dimorphic signals are used in both intrasexual (competition, typically male-male competition) and intersexual (mate choice, typically female mate choice) interactions. Traits traditionally thought to have evolved via competition include those that directly affect the physical competitive ability of males, such as larger body size, antlers, or other armaments, while traits typically associated with mate choice include signals such as coloration or specialized ornaments such as modified feathers that are unlikely to have arisen via natural selection [1]. There is a long-standing expectation that taxa may have multiple sexually dimorphic traits as a result of the separate action of competition and mate choice (e.g., [1]). If intrasexual and intersexual processes are acting independently, we would expect the evolution of dimorphism in a trait classically associated with competition to be unlinked (over evolutionary time and in a given taxa) with the evolution of dimorphism in a signaling trait that might be associated with mate choice.

Although the evolution of a sexually dimorphic trait via competition and a second trait via mate choice may be expected within the same taxon, how can we explain the presence of multiple sexually dimorphic traits that are likely to have evolved through the same selective pressure? In the case of competition, where each individual trait may confer a net competitive advantage (e.g., each type of armament increases male competitive ability in an additive fashion), it would be predicted that multiple traits should accumulate over evolutionary time. In contrast, assessing multiple signals used for intersexual interactions is likely to be costly for female receivers, making it difficult to understand why 
females would bear the cost of assessing multiple signaling traits in mate choice. Thus, the majority of theories to explain multiple sexual signals has developed in the context of mate choice (reviewed in $[2,3]$ ).

Because signals can be costly to assess, produce, and maintain, the development of theory to explain the gain and maintenance of multiple traits used in intersexual signaling has focused on the identification of plausible mechanisms to explain why multiple signals, rather than a single signal, are used by a receiver. Candolin [2] reviewed seven classes of explanations including (1) different messages are conveyed by the different signals, (2) that there are backup or redundant signals, (3) some signals are used primarily in species recognition, (4) some signals are unreliable or Fisherian cues and thus not informative, (5) two signals are received more efficiently than one (receiver psychology), (6) different sensory environments favor different signals, and (7) intersexual conflict/antagonistic coevolution results in a dynamic in which older signals become uninformative antes and newer signals arise. In his more recent review, Bro-Jørgensen [3] also summarizes this theory, albeit slightly differently. In addition to the explanations discussed by Candolin [2], Bro-Jørgensen [3] expands the list to include the rare male effect (i.e., the hypothesis that an unusual signal is always advantageous) and proposes a novel explanation for the advantage of multiple sexual signals: the idea that fluctuating signaling environments favor different signals at different times. Bro-Jørgenson [3] argues that since dynamic social and physical signaling environments are the rule, rather than the exception, this dynamic hypothesis may be a more general explanation for multiple signaling systems than what has been proposed previously.

As can be seen, there is a wealth of plausible hypotheses available to explain the use of multiple sexual signals (Table 1). A critical task at present is to evaluate whether or not there is any dominant mechanism at work across taxa, or whether many different hypotheses will be necessary to understand the ubiquity of multiple sexual signals. The existing hypotheses are framed in a strongly microevolutionary context. As a result, we have detailed predictions about the expression of multiple traits and their use and costs within species (or populations) [2], but much less clarity about the expectations at the macroevolutionary (above species) scale regarding patterns of trait diversification, loss, or the association of traits in lineages where multiple sexual signals occur. However, looking across lineages over evolutionary time should clarify whether there might be one or a few mechanisms, or whether multiple hypotheses are needed to explain the broad patterns seen across taxa.

In an effort to relate this existing (mostly microevolutionary) theory to patterns quantified at the macroevolutionary scale, we have reviewed the major classes of hypotheses available to explain the existence of multiple sexual signals (Table 1) and extended these (when necessary) to make predictions at the macroevolutionary scale. Although competition is expected to lead to the accumulation of traits associated with physical combat over evolutionary time (as described above), we also recognize that intrasexual competition can lead to the evolution of signals that indicate competitive advantage (e.g., [14, 15]). Just as the assessment of multiple signals is likely to be costly for females choosing mates, the assessment of multiple signals indicating competitive advantages in intrasexual interactions are also likely to be costly. Thus, we argue that many of the hypotheses put forward to explain multiple cues in intersexual communication (mate choice) should also apply to the signals used in intrasexual interactions as well (e.g., [16]).

In extending the predictions of the existing theory to the macroevolutionary scale, we made the simplifying assumptions that signals of the same mode (e.g., coloration) are more likely to be redundant in the information they convey, whereas the presence of multiple signals of different modes (e.g., color patterns versus specialized structures) are more likely to communicate multiple different messages or to act in concert. These assumptions can be logically argued and are supported by data (e.g., $[17,18]$ but see $[19]$ ).

The upper portion of Table 1 focuses on the models that deal with signaling in general (applicable to both mate choice and competition), summarizes the major classes of hypotheses to explain multiple sexual signals, and provides our macroevolutionary expectations for sexual dimorphism and the pattern of trait accumulation and loss over time from each of models. In the lower portion of Table 1, we discuss hypotheses that are specific just to mate choice. Specifically, this includes rare male advantages in mate choice [9], antagonistic coevolution in sexual signaling $[10,11]$, and Fisher Runaway processes $[4,12,13]$. These models do not make specific predictions about the types of traits that should evolve but have been framed in a dynamic context and thus make more explicit macroevolutionary predictions than the majority of hypotheses.

To evaluate support for the macroevolutionary predictions of the models, we used a comparative framework to examine the distribution of sexual dimorphism in multiple traits in the family Phasianidae (chickens, pheasants, and allies). Although this is a very broad-brush approach, it has the potential to highlight general patterns and trends. Thus, this can be viewed as the first step for more detailed studies in specific clades that further refine our understanding of the evolution of multiple sexual traits.

\section{Methods}

2.1. Study System. The avian family Phasianidae (within the order Galliformes) contains some of the best-studied avian species, including the domestic chicken (Gallus gallus) and turkey (Meleagris gallopavo), both of which now have complete genomes available $[21,22]$. Species within the family exhibit a wide range of variation in size and external morphology [23]. Species range greatly in size, varying from relatively small ( $\sim 35 \mathrm{~g}$ in Coturnix chinensis) to quite large (up to $5 \mathrm{~kg}$ in the turkey). While many species have little to no sexual size dimorphism, in others males may be more than $30 \%$ larger than females. Some species also exhibit one to several spurs on the tarsi, though these are absent in many species [24]. In some cases, spurs are found in both sexes, while in others they are restricted to males or males have 


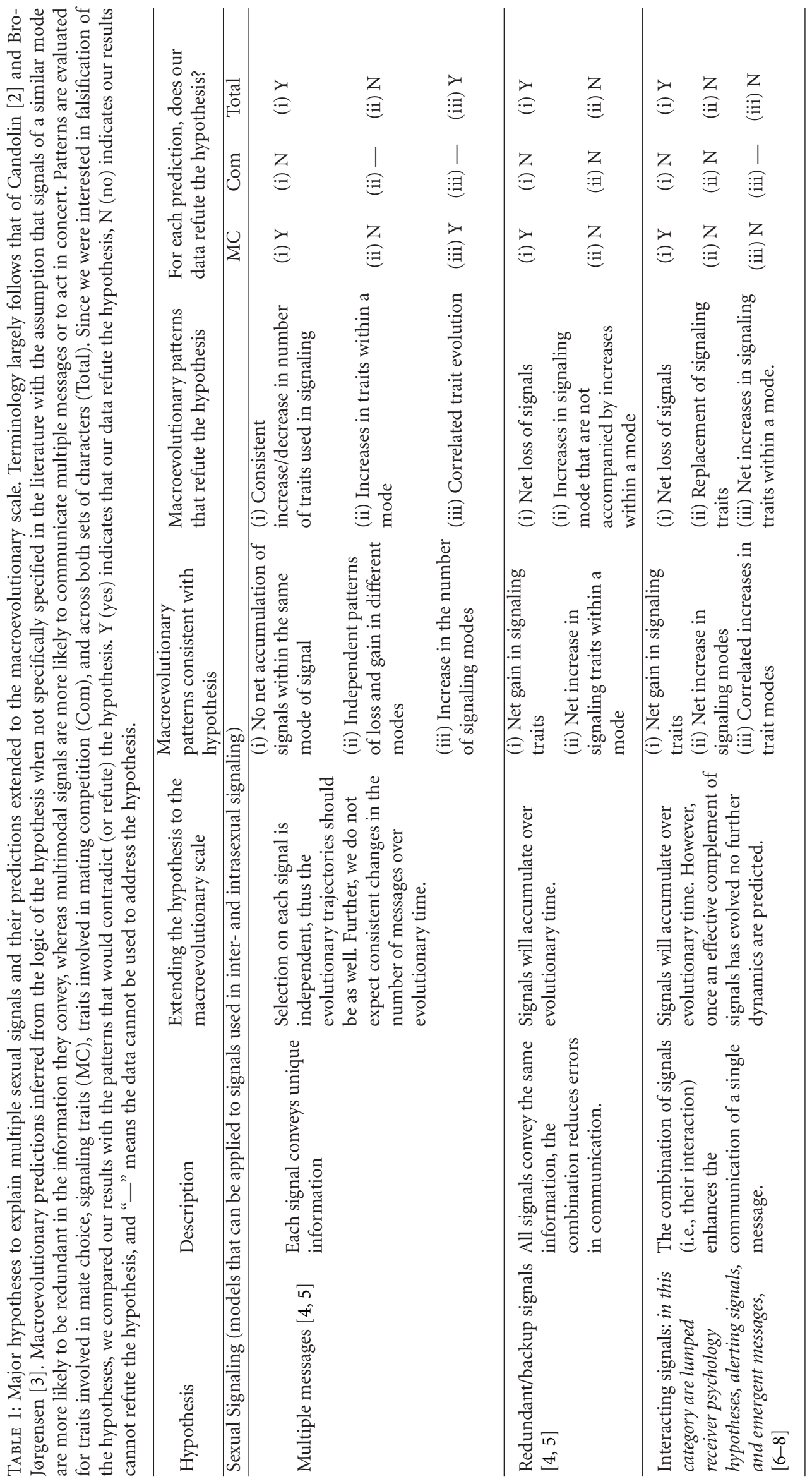




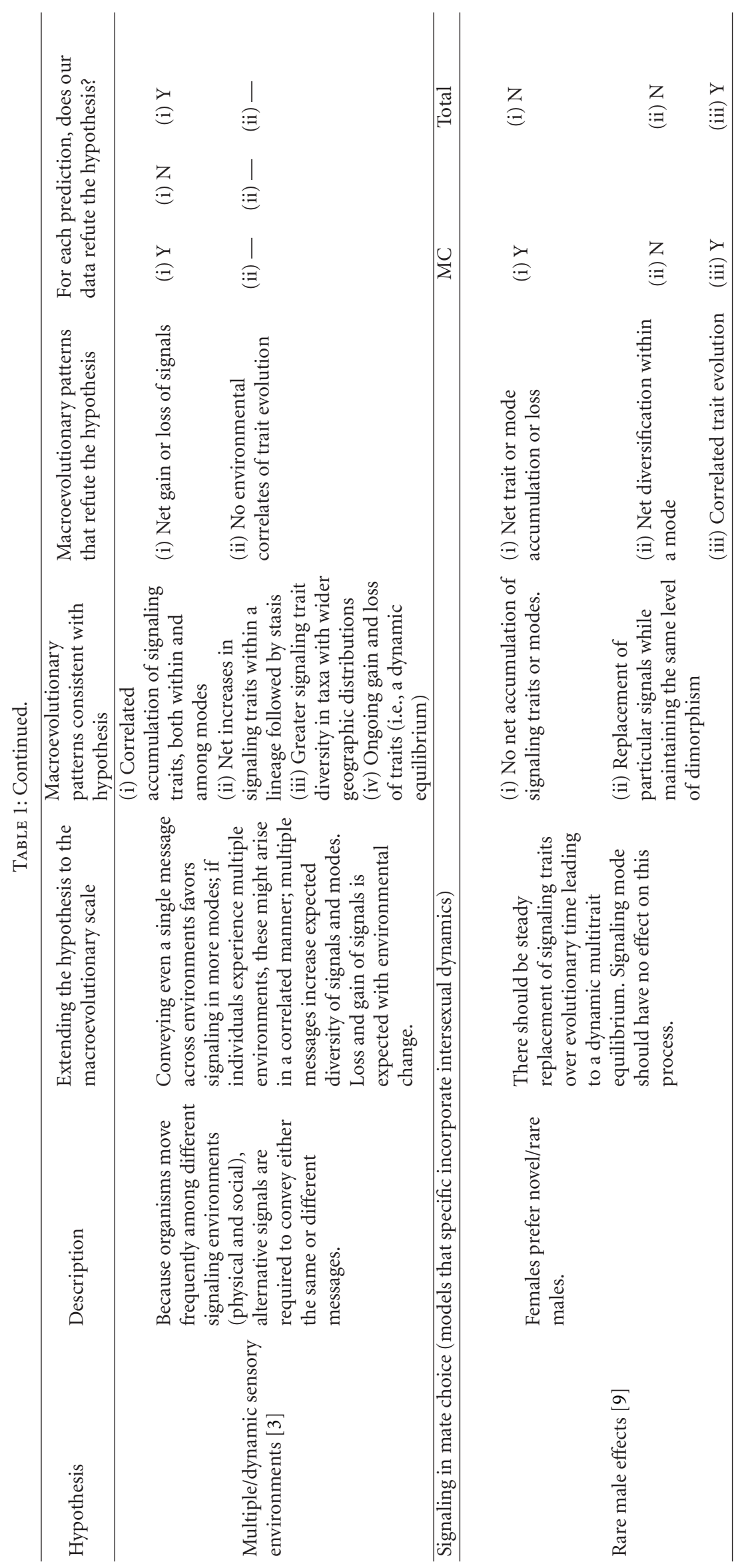




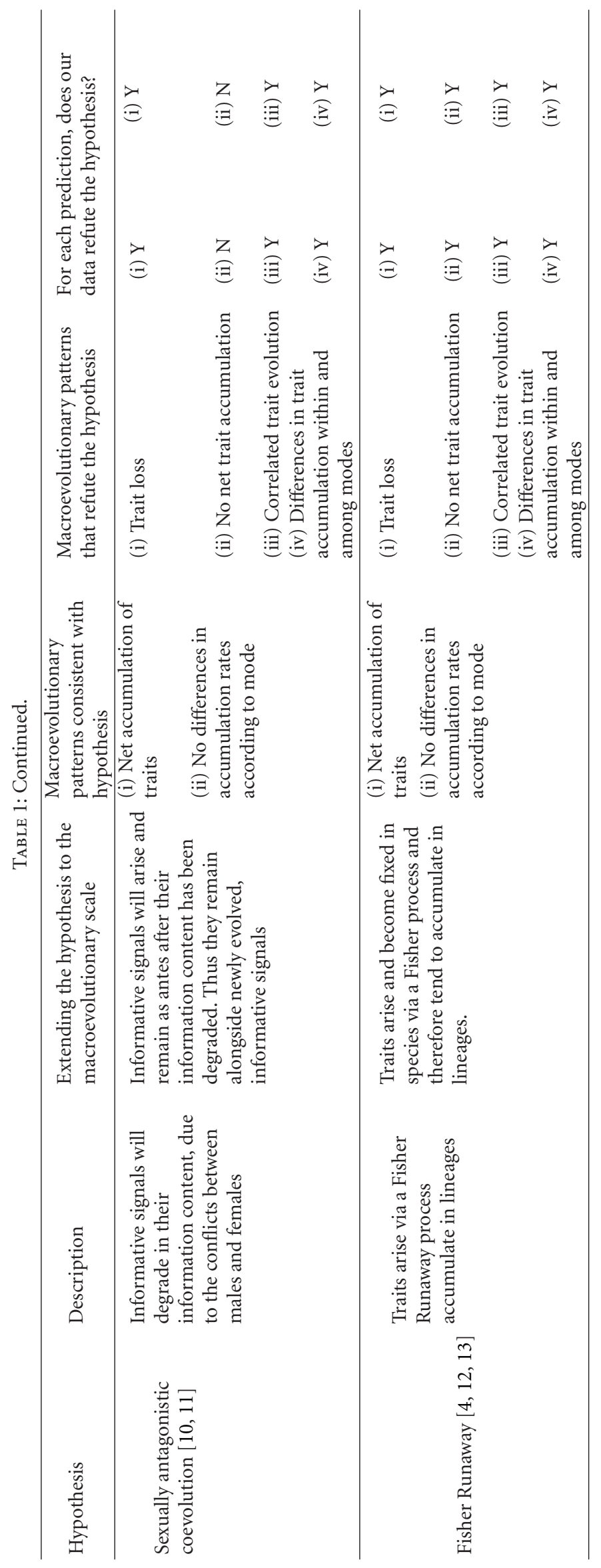


more spurs than females (e.g., females typically have one, while males may have up to three).

Plumage is also quite variable, ranging from species that are cryptically colored with no specialized feathers or extravagant coloration in either sex, to others in which males are strikingly colored, exhibit high levels of iridescence, or have specially modified feathers [23]. Specialized feather types are found in many species, such as greatly elongated or modified tails as well as head modifications such as crests or ear tufts; in some cases these can be found in both sexes, while in others they are either restricted to males or are more exaggerated in males. Additionally, some taxa have very uniquely modified feathers, typically found only in males, such as the highly modified tail coverts of peafowl (Pavo spp.), the elongated hackle and saddle feathers of most junglefowl species (Gallus spp.), or the elongated secondary wing feathers in the argus pheasant (Argusianus argus).

Some species in the Phasianidae (and in galliformes overall) also have regions on the head and neck that lack plumage, instead showing the "flesh" underneath [25]. These fleshy traits can vary from simply bare regions around the eye to the complete absence of feathers on the head and neck of the turkey and specialized structures such as the comb and wattle of junglefowl. In some phasianid species, these fleshy traits have the ability to be "erected" in which the trait can greatly change its size and appearance in a short time, and then be retracted (often being barely visible in the nonerect state). Taxa that are able to rapidly erect these fleshy structures form a clade $[25,26]$, suggesting that the physiological basis for trait erection may have evolved a single time. Fleshy traits are typically found in both sexes when they are present in a species. However, the traits can still be dimorphic, since males often exhibit larger or more brightly colored fleshy regions than females. Moreover, the ability to erect these traits is found only in males.

In part due to the large number of ornamental or exaggerated traits, the role of both competition and mate choice has been extensively studied in several species in this family. Results of these studies have varied and highlight the role of sexual selection in this group. For example, in red junglefowl (the ancestor to domestic chickens, also Gallus gallus), the size of the male comb appears to be most consistently used by females in mating decisions (e.g., [27]), and along with body size, correlates with dominance [28]. Manipulation of social structure indicates that females prefer males with large combs, even when these are not the dominant male [29]. In the Indian peafowl ( $P$. cristatus), females prefer males with more ocelli (eye-like spots) in the modified tail coverts that form the train (e.g., [30, 31]), though this may not be consistent across populations [32]. Both train and tarsus length appeared to be involved in competition in the Indian peafowl [31]. In wild turkey, both competition and mate choice favor males with long snoods $[33,34]$, the fleshy protrusion above the beak. Studies in the ring-necked pheasant (Phasianus colchicus) and various species of grouse have been less clear, but implicate various aspects of morphology and display behaviors in mate choice (e.g., [35-38]).

Early studies on relationships within the Phasianidae showed little consensus (reviewed by $[20,39]$ ). The family exhibits limited osteological variation (e.g., [40, 41]), and the group appears to have undergone a relatively rapid radiation [42], both of which probably contribute to the difficulties associated with resolving phylogenetic relationships. More recent studies using both whole mitochondrial sequences, sequences from multiple nuclear loci, as well as insertions of transposable elements, have resulted in a better-resolved and more stable phylogeny [25, 43-49]. Specifically, these studies agree on several key points. First, the grouse and turkeys, traditionally separated into separate subfamilies or families, nest within the Phasianidae. Second, the traditional grouping of pheasants (dimorphic, highly ornamented, large bodied) and the partridges and old world quail (cryptic, little ornamentation, small bodied) into separate clades within the family is erroneous. Instead, the characteristics that have traditionally been used to define pheasants and to differentiate them from partridges have been suggested to have arisen independently in multiple lineages [42], though this hypothesis has not been tested explicitly.

The suggestion that highly ornamented and dimorphic "pheasants" repeatedly evolved from more monomorphic "partridge" ancestors suggests that studying the Phasianidae may provide insight into the forces that have led to complex patterns of sexual dimorphism and the evolution of multiple male secondary sexual traits. Recently, it has been shown that the evolution of sexual size dimorphism within the Phasianidae may be related to mating system (particularly lekking; [50]), though other types of traits that are potentially involved in sexual selection (e.g., plumage, fleshy traits, and spurs) were not considered. Herein we use a comparative approach to examine the evolution of dimorphism in morphological traits that have been implicated in sexual selection, mapping the gain and loss of dimorphism across the family to generalize about the evolution of multiple sexual signals.

2.2. Phylogenetic Estimation. To obtain a taxon-rich phylogeny, we used a supermatrix analysis (a large-scale data matrix) rather than a supertree analysis (meta-analysis of previous work) $[51,52]$. To ensure all gene partitions were represented by a good range of taxa in the supermatrix, we restricted our analyses to the six data partitions analyzed by Kimball and Braun [25], which included two mitochondrial coding regions (cytochrome $b$ [CYB] and $\mathrm{NADH}$-ubiquinone oxidoreductase chain 2 [ND2]) and four nuclear introns (ovomucoid intron G [OVMintG], $\beta$ fibrinogen intron 7 [FGBint7], pterin-4- $\alpha$-carbinolamine dehydratase intron 3 [PCBD1int3], and rhodopsin intron 1 of [RHOint1]). Kimball and Braun [25] obtained a complete data matrix comprising all of these gene regions for 44 galliformes, representing all galliform families but placing an emphasis on the Phasianidae. For the present study, we extended the taxonomic coverage by retrieving all sequences homologous to the six focal loci in the NCBI database using BLASTN [53] and filtering the sequences to retain a single representative per species. Although the present study focused on Phasianidae, all nonphasianid galliform species that were available for these partitions were included as 
outgroups. CYB and ND2 sequences were equal in length (with the exception of modest variation in the CYB stop codon (see [54]), making them straightforward to align. The nuclear intron sequences were added to the Kimball and Braun [25] alignment and then optimized by eye. We were able to add data to all partitions in the Kimball and Braun [25] matrix. This resulted in a data matrix in which $51.8 \%$ of the cells were filled; all taxa (170 species) were represented by some mitochondrial data and 99 taxa (70 of which were phasianids) also included nuclear intron data.

Maximum likelihood (ML) phylogenetic analyses were performed using the GTRMIX method implemented in RAxML 7.0.4 [55], which performs searches using the GTRCAT [56] method to accommodate among-sites rate heterogeneity followed by a final optimization using the GTR $+\Gamma$ model. ML searches were conducted using 25 distinct randomized maximum parsimony (MP) starting trees. RAxML analyses were conducted both with and without partitioning the data. Partitioned analyses were conducted using 10 data partitions, one for each nuclear intron and three for each of the mitochondrial coding regions (one partition for each of the codon positions). Bootstrap analyses used the GTRCAT method and 500 replicates.

To accommodate uncertainty in phylogenetic reconstruction when examining patterns of character change, we used MrBayes 3.1 $[57,58]$ to generate a set of trees that accommodates the uncertainty in both topology and branch lengths. For these analyses, the data were partitioned as described above for RAxML. For each partition, we used the AIC to select the best fitting model that is implemented in MrBayes. We ran four chains (three of which were heated) for $7.5 \times 10^{7}$ generations and discarded the first $1 \times 10^{7}$ generations. We sampled every $1 \times 10^{4}$ generations, resulting in a set of 6500 trees that were used for trait reconstruction (see below).

2.3. Trait Coding and Analyses. The focus of this study was to examine gains and losses in sexual dimorphism, so all traits were coded as binary characters, where $0=$ monomorphic and $1=$ dimorphic. Thus, a monomorphic score $(0)$ could mean a trait was absent in both sexes or present and similar in both sexes; a dimorphic score (1) could mean either that the trait was present in both sexes but larger or modified in one sex or present in one sex and absent in the other. Most information used to code traits was obtained from Madge and McGowan [23], though this source was supplemented with information in Johnsgard [59-61]. Although the Phasianidae were the focus of this study, we also coded data for the five species of guineafowl (Numididae) and six available New World quail (Odontophoridae) represented in the sequence dataset to polarize traits.

A total of nine binary characters were scored for dimorphism (Table 2). Five of these corresponded to plumage differences: (1) plumage color, (2) tail length, (3) head ornaments (e.g., crests and ear tufts), (4) other plumage differences (e.g., iridescence, unusual plumage features not covered in other traits), and (5) whether the male plumage was highly elaborated compared to females. The remaining four traits included (6) fleshy traits, (7) spurs, (8) wing size, and (9) overall body size. Body size is difficult to measure, and we examined several possibilities. First, we looked at a qualitative difference based on descriptions in Madge and McGowan [23]. Second, we took weight measurements for males and females from Madge and McGowan [23], although some of these were based on single individuals and/or captives whose weights may not be typical. Third, we considered the weights from Lislevand et al. [50], which included values that were based on three or more individuals, primarily measured during the breeding season. For the two weight measures, we considered species dimorphic if there was at least $10 \%$ difference between the sexes $[20,50]$. Since the patterns using weight data were similar to those using qualitative measurements (including in the relative differences between loss and gains), but were for many fewer species, we only report those results using the first approach.

Several additional characters were generated from this initial set, as defined in Table 2. We also scored whether species were dimorphic in any plumage trait (10) and dimorphic in any competitive trait (11). To highlight species that exhibited strong dimorphism in either plumage or competitive traits, species were scored for two additional binary characters: (12) high plumage dimorphism and (13) high competitive dimorphism. To look at overall patterns, we also made several composite traits, including totaldimorphism (character 14; sum of the state of characters 1-9), total signal dimorphism (character 15; sum of characters 1-6), total competitive dimorphism (character 16; sum of characters 7-9), and total plumage dimorphism (character 17; sum of characters 1-5).

To assess overall rates of change and relative rates of gains and losses, we used maximum likelihood reconstruction in BayesTraits [62] using the 6500 phylogenetic trees from our Bayesian analysis (above). From this, we estimated the transition rate and the bias (the ratio of the gain rate to the loss rate) for each binary character. Although the ML estimates obtained using this procedure do not represent posterior distributions in the Bayesian sense, this approach captures the impact of phylogenetic uncertainty on our estimates and it has the advantage of being independent of priors. We report the median estimate as well as the top 2.5 percentile and the bottom 2.5 percentile of the distribution (95\% range) for both the rate and bias. To determine whether there was a significant difference between gains and losses in the binary characters (1-13), we used Mesquite [63] and the ML tree from RAxML to test whether a two-rate model (gains $\neq$ losses; AsymmMk) was significantly better than a one-rate model (gains $=$ losses) with a likelihood ratio test, assuming that twice the difference between models in their $\log$ likelihood is $\chi^{2}$ distributed with 1 degree of freedom.

To understand the overall patterns in the ancestor, we used the output from BayesTraits [62] to assess the probability of being dimorphic for characters 1 to 9 . We did this at three possible ancestral states: (1) the ancestor of the outgroup taxa (Numididae and Odontophoridae) and the Phasianidae, (2) the ancestor to the Phasianidae, and (3) the 
TABLE 2: Characters scored as monomorphic or dimorphic.

\begin{tabular}{|c|c|c|}
\hline & Character & Description \\
\hline 1 & Plumage-Color & $\begin{array}{l}\text { Dimorphic scored for obvious differences between the sexes in coloration (subtle differences, such as } \\
\text { slightly brighter in males or more extensive spotting in one sex were scored as monomorphic) }\end{array}$ \\
\hline 2 & Plumage-Tail Length & Dimorphic scored when tail (or tail coverts) was much longer or modified in males relative to female tail \\
\hline 3 & $\begin{array}{l}\text { Plumage-Head } \\
\text { Ornaments }\end{array}$ & Dimorphic scored if ear tufts, crests, or capes either present in males or larger in males \\
\hline 4 & Plumage-Other & $\begin{array}{l}\text { Dimorphic scored if male plumage exhibits iridescence or modified feathers not covered in other } \\
\text { categories }\end{array}$ \\
\hline 5 & Plumage-Elaboration & $\begin{array}{l}\text { Dimorphic scored if the male plumage pattern or color is dramatic and very obvious relative to female } \\
\text { plumage }\end{array}$ \\
\hline 6 & Fleshy Traits & $\begin{array}{l}\text { Dimorphic scored if one sex larger or obviously brighter than the other; since the goal was to explore } \\
\text { sexual selection on these traits, the appearance of the trait when displayed was scored }\end{array}$ \\
\hline 7 & Spurs & $\begin{array}{l}\text { Dimorphic scored if males had more spurs than females, or in a few cases male spurs noticeably longer } \\
\text { than female spurs }\end{array}$ \\
\hline 8 & Wing Length & $\begin{array}{l}\text { Dimorphic if at least a 5\% difference between the sexes (following Höglund [20]). Used average wing } \\
\text { length if given; if only a range was given, then the midpoint of the range was used for calculations) }\end{array}$ \\
\hline 9 & Body Size & $\begin{array}{l}\text { Dimorphic if species noted as having larger males or smaller females; scored as monomorphic if } \\
\text { differences were noted as slight (weight was not used as many measures in the literature taken from } \\
\text { captive individuals and/or dead specimens whose weight may not be representative) }\end{array}$ \\
\hline 10 & Any Plumage & Scored as one if dimorphic in any of the five plumage traits \\
\hline 11 & Any Competitive & Scored as one if dimorphic in either spurs, wing length, or body size \\
\hline 12 & High Plumage & Scored as one if the species exhibited dimorphism in at least three of the five plumage characters \\
\hline 13 & High Competitive & Scored as one if the species exhibited dimorphism in at least two of the three competitive characters \\
\hline 14 & Total & Sum of all characters scored as dimorphic in a species \\
\hline 15 & Total Signal & Sum of the plumage and fleshy trait dimorphism, both of which are used in visual displays \\
\hline 16 & Total Competitive & Sum of spur, wing, and body size characters scored as dimorphic \\
\hline 17 & Total Plumage & Sum of the five plumage characters scored as dimorphic \\
\hline
\end{tabular}

ancestor to the core Phasianidae (excluding the Arborophilinae from the remaining phasianids, Figure 1). For each trait, we summed the probability of being dimorphic for all nine characters (character 14), total signal dimorphism (character 15), total competitive (character 16), and total plumage (character 17) across all trees. We then calculated the median value and the $95 \%$ range.

To determine whether changes in one type of trait (e.g., plumage) were associated with transitions in another (e.g., fleshy traits), we looked for correlations using correlated changes [65] as implemented in Mesquite 2.72 [63]. For each test, we used 10 likelihood iterations and ran 100 simulations to establish the distribution. We ran this test with several combinations of traits. First, we examined whether there was a relationship between having dimorphism in any plumage trait (10) with any competitive trait (11). We also tested all pairwise combinations of high plumage dimorphism (12), high competitive dimorphism (13), and fleshy (6) characters to examine whether there was an association between being highly dimorphic in two different types of traits.

\section{Results and Discussion}

3.1. Phylogeny. The combined alignment of 170 taxa (127 phasianids) was $6296 \mathrm{bp}$ in length, of which $2184 \mathrm{bp}$ were mitochondrial and 4112 bp were nuclear. The nuclear intron data included some regions that were difficult to align or present only in a small number of taxa (e.g., the mountain quail (Oreortyx pictus) had an autapomorphic insertion that corresponded to a 579-bp segment of an endogenous retrovirus $(\mathrm{ERV}))$. The nuclear intron data also included two microinversions. None of the rare genomic changes (the ERV insertion and microinversions) united controversial groups: two were autapomorphic and one united the genus Gallus. After the difficult-to-align regions and rare genomic changes were excluded from analyses, there were 2828 bp of nuclear intron data available for the phylogenetic analyses. For the data that were analyzed, there were 1298 variable mitochondrial sites (1159 were parsimony-informative) and there were 1877 variable nuclear intron sites (1451 were parsimony-informative).

Our analyses of the supermatrix yielded trees (Figure 1) that were more similar to other recent analyses of galliform phylogeny based upon sequence data (e.g., $[25,26,43,44,46,49,66,67])$ than to the available galliform supertrees $[68,69]$. Points of agreement between the supermatrix trees and other studies include the strong support for dividing Galliformes into five major clades: Megapodidae (megapodes), Cracidae (chachalacas, guans, and curassows), Numididae (guineafowl), Odontophoridae (New World quail), and Phasianidae (pheasants and partridges). Relationships among these groups (especially the position of the New World quail) are variable in supertrees 

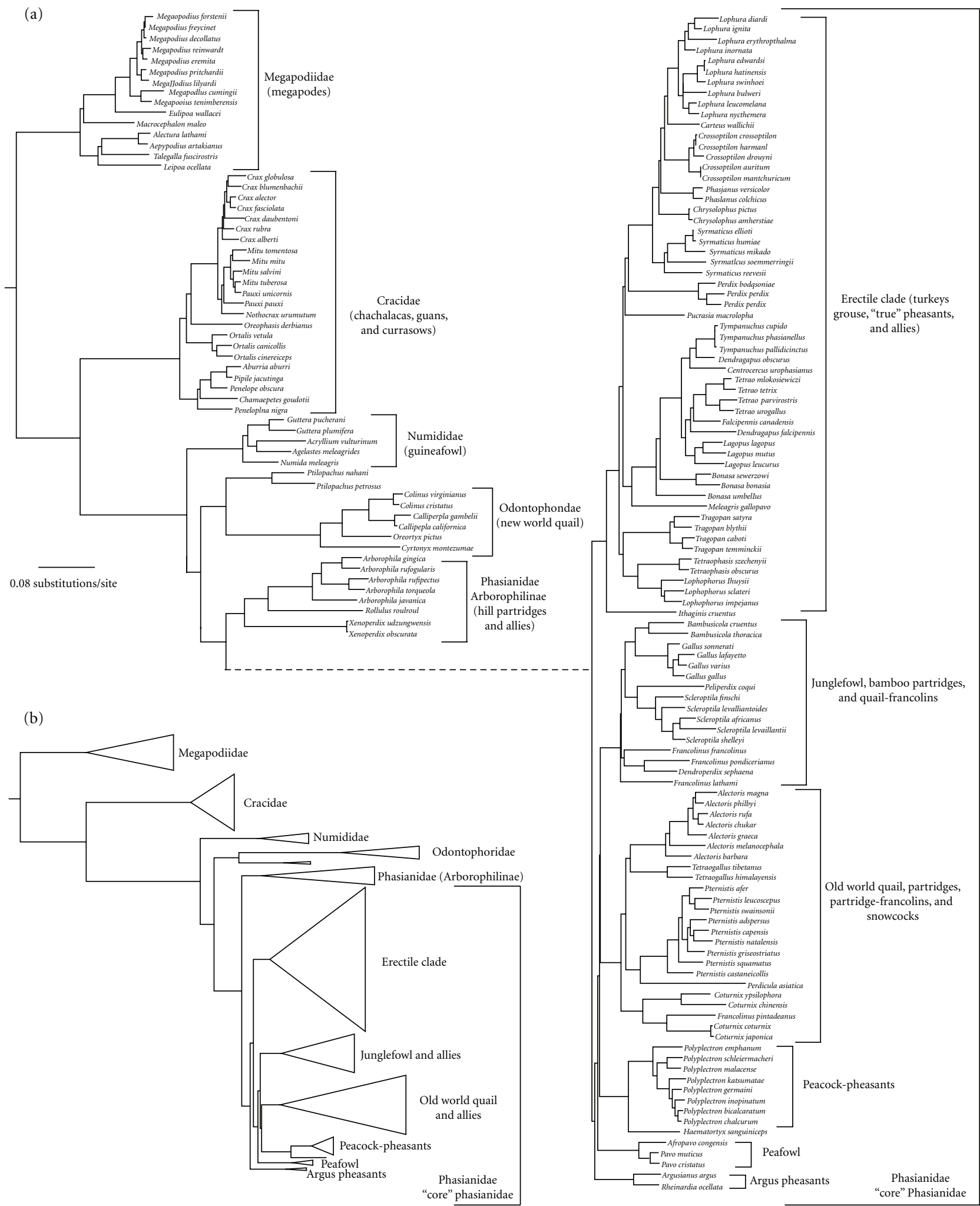

FIGURE 1: Supermatrix phylogeny of Galliformes. Estimates of phylogeny for galliformes were obtained by unpartitioned (a) and partitioned (b) ML analyses. The root of Galliformes was placed between Megapodidae and other galliformes, a position consistent with large-scale studies that included both outgroups and members of all five families $[43,64]$. Stars indicate that the adjacent branch had strong $(\geq 95 \%)$ bootstrap support, whereas dots nodes indicate that the adjacent branch had moderate $(\geq 70 \%)$ bootstrap support. The topology and degree of support for the partitioned Bayesian MCMC analysis were virtually identical to that of the partitioned ML analysis (if the tendency for Bayesian posterior probabilities to exceed ML bootstrap values is considered). 
TABle 3: Patterns of evolutionary change using values estimated from BayesTraits. * indicates traits where there was a significantly better fit to a two-rate model than a one-rate model.

\begin{tabular}{llcccc}
\hline & & Median rate & 95\% Range for rate & Median gains/loss & 95\% Range for gains/loss \\
\hline 1 & Plumage-Color & 1.65 & $1.32-2.11$ & 0.78 & $0.45-1.00$ \\
2 & Plumage-Tail Length & 1.34 & $1.04-1.57$ & 0.26 & $0.17-0.42$ \\
3 & Plumage-Head Ornaments & 3.48 & $2.73-4.36$ & 0.25 & $0.21-0.33^{*}$ \\
4 & Plumage-Other & 1.51 & $1.20-1.88$ & 0.17 & $0.11-0.23^{*}$ \\
5 & Plumage-Elaboration & 2.41 & $1.87-3.49$ & 0.22 & $0.17-0.31^{*}$ \\
6 & Fleshy Traits & 0.88 & $0.57-1.08$ & 0.57 & $0.26-1.38$ \\
7 & Spurs & 0.39 & $0.32-0.48$ & 0.84 & $0.32-1.77$ \\
8 & Wing Length & 18.40 & $3.68-891.1$ & 1.21 & $0.77-1.26$ \\
9 & Body Size & 1.67 & $1.13-2.36$ & 1.55 & $0.83-2.43$ \\
10 & Any Plumage & 1.54 & $1.10-1.79$ & 0.92 & $0.41-1.21$ \\
11 & Any Competitive & 0.92 & $0.79-1.18$ & 0.14 & $4.75-9.57$ \\
12 & High Plumage & 1.71 & $1.36-2.07$ & 1.25 & $0.10-0.24 *$ \\
13 & High Competitive & 2.08 & $1.78-2.90$ & $0.93-1.81$ \\
\hline
\end{tabular}

$[68,69]$ but relationships among these clades in our tree are consistent with studies based upon independent gene regions $[25,44,46,67]$, and the combination of both morphological and molecular data [43].

The deepest divergence within the Phasianidae was between two well-supported clades, the Arborophilinae [43] and a large "core phasianid" clade. Arborophilinae includes hill partridges (Arborphilia spp.), crested wood-partridge (Rollulus roulroul), and recently discovered African forest partridges (Xenoperdix spp.), consistent with results from total evidence studies [43]. The core phasianid clade included the strongly supported "erectile clade," which includes a number of taxa that are able to rapidly erect fleshy traits $[25,26]$. The erectile clade has also been found in other recent studies $[43,67]$. The majority of the remaining core phasianids form a clade in both analyses presented here, with the exception of the argus pheasants (Argusianus argus and Rheinardia ocellata) which form the sister group of the remaining core phasianids in the partitioned analysis. This agreement with prior estimates of galliform phylogeny (e.g., $[25,43,49,67])$ indicates the trees are sufficiently accurate to allow rigorous examination of the evolution of sexual dimorphism in this order.

Despite the congruence between our supermatrix trees and prior estimates of galliform phylogeny, the differences between trees obtained with and without partitioning (Figure 1) raise the question of whether the core phasianids can be divided into two large clades (i.e., the erectile clade and a second clade that comprises the remaining core phasianids) or three clades (the erectile clade, the argus pheasants, and the remaining core phasianids). Recent phylogenetic studies of galliformes (e.g., [25, 26, 43, 47-49, 67]) have reached contrasting conclusions regarding this question, but there is no consistency among studies. Moreover, the relevant branches are short, and support for the conflicting relationships is limited both here and in previous studies. Given this region of conflict, we mapped traits on both trees (Figure 1), though only the results from the unpartitioned tree are shown (using the partitioned tree yielded similar results).
3.2. Character Evolution. As can be seen when looking at total dimorphism (character 14), species that are highly dimorphic (e.g., dimorphic in seven or more characters) can be found throughout the Phasianidae (Figure 2). While some clades are comprised of species that are generally highly dimorphic, and others of species that exhibit little dimorphism, there are several cases in which taxa with relatively high levels of dimorphism are sister to taxa that have very low levels of dimorphism. As expected given this level of variation throughout the family, all nine dimorphism characters exhibit both gains and losses, and many are evolving at relatively high rates (Table 3 ). The lowest rate of change occurs in spur dimorphism, with the other traits typically exhibiting rates of change that are between 2- to 10fold greater than for spur dimorphism (although wing length shows a much greater relative rate of change, topological uncertainty had a relatively large impact upon the estimates on this character, and the $95 \%$ range is so broad, it is difficult to compare this to the other characters).

Although all characters exhibited gains and losses, the relative rate of gains and losses differed among characters (Table 3). Signal traits (plumage and fleshy traits) exhibited a greater rate of loss than gain (gain/loss values $<1$ ), while most competitive characters showed a greater rate of gains than losses (the exception is spurs, where the median value is near one, indicating the rate of gains is similar to that of losses). However, in most cases, the difference between the rate of gains and losses was not substantial, and the estimated 95\% range of gains to losses across the Bayesian trees frequently included one. Although the 95\% ranges that we report reflect the impact of topological uncertainty upon the ML estimate (not the confidence interval on the ML estimate given a fixed topology), the results based upon the 95\% range were typically consistent with the results of the likelihood ratio test when it was used to select the best fitting model. All traits with a 95\% range that included one did not show a significantly better fit to a two-rate model (gains and losses occur at different rates) relative to a one-rate model (equal gain and loss rates). In contrast, many traits in which the $95 \%$ 


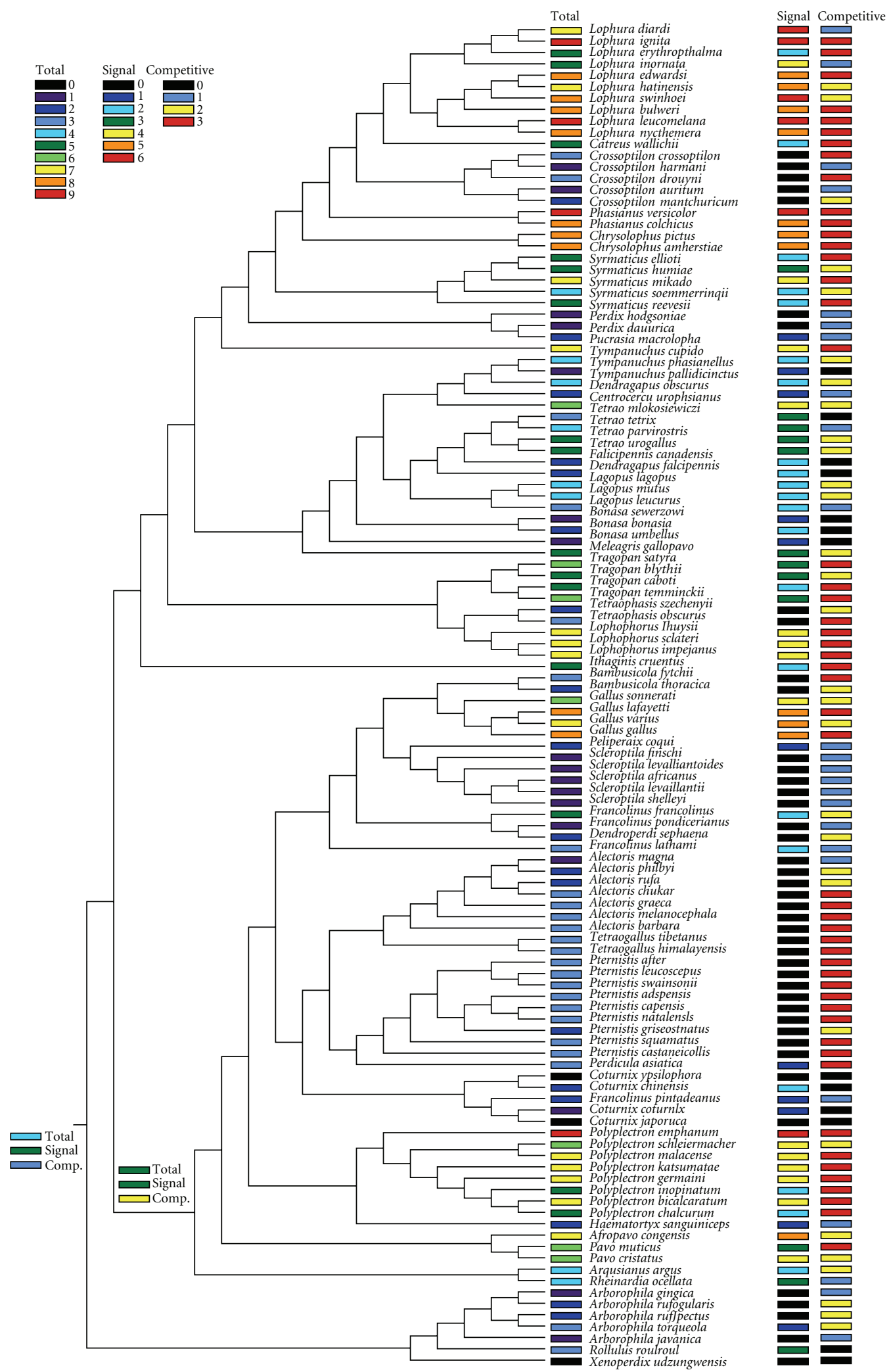

Figure 2: Patterns of evolution for sexual dimorphism in the Phasianidae. Values for total dimorphism (character 14), total signal dimorphism (character 15), and total competitive dimorphism (character 16) in extant taxa are presented to the right of the unpartitioned ML topology. The estimated ancestral states for these dimorphism characters are presented for the Phasianidae and the core Phasianidae. Ancestral state estimates reflect the median of the set of ML estimates obtained using the trees sampled from the Bayesian MCMC analysis. 
range did not include one showed a significantly better fit to a two-rate model (Table 3; note that the likelihood ratio test for character 2 approached a significant improvement with a two-rate model; $2 \Delta \log$ likelihood $=3.70,2 \Delta_{\text {crit }}=3.84$, $\mathrm{df}=1, P=.054)$.

Directional selection for continued elaboration of sexually dimorphic traits is assumed in many models of sexual selection (e.g., [1]), suggesting that loss of dimorphism should be rare. However, more recent studies have recognized that loss of dimorphism can occur $[66,70]$. Our study further emphasizes this point, as the rate of losses exceeded gains in most of the nine dimorphism characters (Table 3 ). Moreover, even for characters where the rate of gains exceeded that of losses (i.e., characters 8 and 9), the rate for gains was less than twice that of losses. In contrast, the rate of losses was three to four times greater than that of gains for the several of the other characters (i.e., characters 2 through 5).

This pattern of loss suggests that gains in dimorphism likely occurred early in the evolution of the Phasianidae (or earlier), making it more likely that subsequent transitions were losses rather than gains. The summed probability of being dimorphic for all nine characters was low in the common ancestor of Numididae, Odontophoridae, and Phasianidae (Table 4), but increases in the common ancestor of the Phasianidae and is even larger in the ancestor to the core phasianids (Table 4, Figure 2). Similar patterns are seen when summing the signal and competitive traits separately (Table 4, Figure 2), suggesting this early gain of dimorphism involved both types of traits. These results suggest that the characteristics that have traditionally been used to define pheasants and differentiate them from partridges may not have arisen independently in multiple lineages, as suggested by Kimball et al. [42]. Instead, the common ancestor of the core phasianids may have been a dimorphic and "pheasantlike," with the partridge lineages arising multiple times due to the loss of dimorphism.

The only specific traits for which there is a low $(<0.05)$ probability that the ancestor to all phasianids was dimorphic were fleshy traits (character 6) and spurs (character 7). At the base of the core phasianids, only dimorphism in fleshy traits appears very unlikely, while the state for spurs is more equivocal (probability of dimorphism is 0.39 ). The absence of dimorphism in fleshy traits is consistent with hypotheses that fleshy traits evolved through natural selection as a mechanism of thermoregulation in both sexes of Galliformes (and thus are present but monomorphic in many basal lineages), and only in some of the Phasianidae were these coopted for sexual selection $[25,71]$ leading to the evolution of dimorphism for this trait.

To assess whether the pattern of transitions to and from a monomorphic state are similar to the patterns inferred using individual characters, we scored the presence or absence of any plumage dimorphism (character 10). Patterns of gains and losses of dimorphism in any plumage character exhibit roughly equal rates of gains and losses, in contrast to the pattern seen with the individual plumage characters that generally show a higher rate of losses (Table 3 ). The common ancestor to the core phasianids has a relatively high summed probability of dimorphism in the five individual plumage characters (Table 4), making it likely the ancestor that had already gained multiple plumage characters. Under this scenario, any plumage character can only transition to monomorphism if all of the individual dimorphic characters are loss, whereas gain only requires a change in one trait. Thus, loss of dimorphism in any plumage is expected to be much less common for this character than in the individual characters, as we observe.

A very different pattern is seen when birds are scored for the presence of dimorphism in any competitive trait (character 11), where gains appear much more common than losses (Table 3). Although the rate of gain is greater than that of loss, a one-rate model (equal gains and losses) could not be rejected for this character $(2 \Delta \log$ likelihood $=0.2$; $2 \Delta_{\text {crit }}=3.84$, df $\left.=1, P=.66\right)$. This suggests that the likelihood surface for this character is very flat, such that very different gain to loss ratios should result in only minor differences in likelihood values. Since an equal rate of gains to losses could not be rejected it remains possible that the gain to loss ratio is actually much lower than estimates obtained using the trees sampled by the Bayesian analysis (Table 3), making it difficult to interpret patterns of changes in this character at this time.

Some species exhibit more extreme dimorphism, being dimorphic for multiple traits. We examined transitions to and from high degrees of dimorphism in same type of trait. Species that exhibited high plumage dimorphism relative to other species (character 12) showed a strong bias toward loss of dimorphism (Table 3 ). This pattern would be expected given the greater rate of loss for the individual plumage characters and the relatively high probability of plumage dimorphism in multiple traits in the ancestor to the phasianids (Table 4). Similarly, as expected from the patterns observed in individual competitive traits, species that exhibited high competitive dimorphism (character 13) showed the opposite pattern, with gains exceeding losses in extreme dimorphism (Table 3).

Strong sexual selection could select for increased dimorphism in two types of traits (i.e., one signal and one competitive trait). An examination of the overall level of dimorphism in signal and competitive characters indicates that there are clades that are highly dimorphic for both types of traits, clades that exhibit little dimorphism in both types of traits, or clades that are highly dimorphic in one, but not both, types of traits (Figure 2). Thus, there is not a strong, consistent pattern that emerges at this broad level. However, there do appear to be some correlation among transitions in the characters (Table 5) suggesting that the transitions among character states may not be independent of other characters. For example, gains in the any plumage (character 10) and any competitive (character 11) are correlated with fleshy traits (character 6), while being highly dimorphic for plumage (character 12) appears to lead to more gains in both fleshy traits (character 6) and being highly dimorphic for competitive traits (character 13).

In summary, the patterns we observed are complex and suggest that no single dominant explanation will be sufficient to explain our observations. Both monomorphism 
TABle 4: Reconstructed dimorphism at ancestral nodes. Ancestral values are the summed dimorphism at each node, followed by the 95\% range in parentheses.

\begin{tabular}{lcccc}
\hline & Maximum possible & Ancestor of outgroups & Ancestor of Phasianidae & Ancestor of core phasianids \\
\hline Total dimorphism (14) & 9 & $2.8(1.7-3.4)$ & $4.0(2.4-5.3)$ & $5.4(4.6-7.1)$ \\
Signal dimorphism (15) & 6 & $1.8(0.4-2.3)$ & $2.7(1.5-3.6)$ & $3.3(2.6-4.4)$ \\
Competitive dimorphism (16) & 3 & $1.0(0.7-1.9)$ & $1.3(0.8-1.8)$ & $2.1(1.6-2.9)$ \\
Plumage dimorphism (17) & 5 & $1.8(0.4-2.2)$ & $2.7(1.5-3.6)$ & $3.3(2.6-4.4)$ \\
\hline
\end{tabular}

TABLE 5: Correlated patterns of change between characters (character number indicated in parentheses). ${ }^{*}$ indicates $P<.05, * *$ indicates $P<.01$.

\begin{tabular}{llcl}
\hline & & $\Delta \log$ likelihood & Highest transition \\
\hline Any plumage (10) & Any competitive (11) & $9.47^{* *}$ & Any plum. $0 \rightarrow 1$, if Any comp. $=0$ \\
Fleshy traits (6) & Any plumage (10) & $11.62^{* *}$ & Any plum. $0 \rightarrow 1$, if Fleshy $=1$ \\
Fleshy traits (6) & Any competitive (11) & $5.31^{*}$ & Any comp. $0 \rightarrow 1$, if Fleshy $=1$ \\
High Plumage (12) & High competitive (13) & $5.39^{* *}$ & High comp. $0 \rightarrow 1$, if High plum. $=1$ \\
Fleshy traits (6) & High plumage (12) & $4.97^{* *}$ & Fleshy $0 \rightarrow 1$, if High plum. $=1$ \\
Fleshy traits (6) & High competitive (13) & 3.77 & No relationship \\
\hline
\end{tabular}

and dimorphism evolved multiple times for all characters (Figure 2 and Table 3). When all traits were considered together there was no clear trend towards gain or loss, although there were some weaker patterns. Consistent with the hypothesis that gains in dimorphism in traits that are directly involved in competition are expected, competitive characters exhibited a larger number of gains than signaling characters. Surprisingly, much of the gain in signal character dimorphism appears in the ancestor to modern phasianids (Figure 2) and losses predominate for these characters within the group (Table 3). This is in contrast both to expectation (e.g., [1]) and our previous hypothesis for the Phasianidae [42].

\subsubsection{Observed versus Predicted Patterns of Character Evolu-} tion. In Table 1, we summarize the patterns we identified that are either inconsistent with our predictions given specific models (and would therefore falsify the model) or consistent with our predictions. It is immediately obvious by reference to Table 1 that there was no single model that was consistently supported that also could not be refuted by our results. Falsification of many models was due to our observed pattern of loss of dimorphism. The overall pattern of trait loss from an early and highly dimorphic ancestor or set of ancestors is one that has not been strongly considered in the field of sexual selection (despite other studies that have highlighted the importance of loss of sexually dimorphic traits $[66,70])$. Since the sexual selection literature has driven the development of hypotheses to explain the presence of multiple sexual signals, loss of signaling traits has not been explicitly considered in these models as well. Thus, these results highlight the need for a more balanced treatment of trait loss and gain in this area. Furthermore, the diversity of observed patterns of gain and loss of dimorphism among lineages within the Phasianidae suggests that the processes affecting the evolution of multiple signaling systems in any single lineage are either temporally variable or diverse. Thus, there appears to be no single, predominant mechanism to explain the evolution of multiple sexual signals.

That said, our test of the multiple/dynamic sensory environments hypothesis [3], essentially the idea that fluctuating signaling environments may favor different signals at different times, is particularly weak. This hypothesis has the potential to be more general than the other hypotheses tested, due to its flexibility and the wide array of patterns it can explain as a result. Within the hypothesis are nested the possibilities that different aspects of a mate or competitor are important in different contexts (i.e., the multiple messages hypothesis) and that different environments may demand different signals to communicate the same message (i.e., receiver psychology). Thus, it is also more comprehensive than other proposed models, as well as being more dynamic. However, adequate tests of this model demand a phylogenetic approach that considers environmental variables alongside signaling traits. Without those comparisons, we cannot seriously evaluate whether this more general model could explain the type of patterns we observed, and such analyses should be conducted in the future.

In contrast, those models for which the phylogenetic predictions are best specified, that is, the models that specifically incorporate interspecific dynamics (bottom portion of Table 1), have little support across the Phasianidae. Thus, none of these models appears to be sufficiently general so as to explain the distribution of multiple sexual signals within this family. This is true, whether we focus exclusively on those characters that are most likely to be the result of mate choice or also include characters that are associated with competitive ability (which females often consider in mate choice; reviewed in [14]).

The remaining hypotheses that we examined are both supported and refuted across the Phasianidae. Thus, even within this one family, the diversity of factors contributing to the evolution and maintenance of multiple sexual signals across taxa appears to be greater than postulated by any single model. However, it is also not possible to exclude any 
of these models as potential explanations for the patterns in at least some lineages within the Phasianidae. The difficulty in excluding any hypothesis may reflect one of two factors. First, it may be that all of these processes contribute to the evolution of multiple signaling traits in this family to at least some degree, though the relative importance of each may vary across taxa. Alternatively, this may also reflect that the macroevolutionary predictions of each hypothesis are currently too imprecise to exclude some models. Additional refinement of the predictions each model makes over evolutionary time might allow the exclusion of some of these in the future.

Taken together, this comparison of data with models to explain the evolution of multiple sexual signals suggests we need to focus on (1) a more explicit consideration of trait loss in the context of sexual selection and the evolution of multiple sexual signals and (2) formally extending the existing models on the evolution of multiple sexual signals to the macroevolutionary scale and thus, strengthening our predictions. Our observed pattern of diversification and losses of multitrait dimorphism in the Phasianidae highlights the complexity of testing among the existing models, particularly as the models are currently described. Additionally, it is clear we need to incorporate environmental characters into these types of analysis to fully test existing models. Finally, to better relate theoretical predictions about the functionality of signaling and competitive traits with their resulting phylogenetic distribution we also need to better understand the costs of different signal types to both signalers and receivers (e.g., [72]), the effectiveness of different types of signals across environments, and the nature of interactions amongst signals.

3.3. Broader Implications and Future Directions. Sexual selection is hypothesized to have led to rapid evolutionary change [1]. If this is true, there are several expectations that should be apparent in a study such as this. First, there may be gains of multiple sexual signals along a single branch in a phylogenetic tree, rather than a slow stepwise gain of such traits at the time that the strength of sexual selection increased. Second, a shift to increasing sexual selection in a lineage may drive rapid radiations and lead to short internodes shortly after that period. Both of these patterns are consistent with our observations in the Phasianidae. The base of the core phasianids contains may short branches (e.g., Figure 1), and it is at this time that it appears that there was a gain in multiple sexually dimorphic traits. Understanding whether this is a general phenomenon or a pattern specific to the Phasianidae will require looking for similar associations between rapid evolutionary radiation and the gain of multiple sexually dimorphic traits in other groups. Additionally, the potential to evolve sexual dimorphism in suites of traits in a short time period may depend on the genetic basis of such traits, which is likely to be complex. To understand the specific genetic changes responsible for sexually dimorphic characters is likely to require combining analyses like these with experimentation in specific species, but should lead to a greater knowledge of the processes that may lead to multiple sexual signals.

Many models for the evolution of sexual signals have been phrased, either explicitly or implicitly, in a microevolutionary framework (see Table 1). This has led to tests using behavioral assays within species focused on assessing the strength and nature of sexual selection. These types of studies are clearly important, but another area that will be important to explore in the future is the development of explicit mathematical models that can be used to examine patterns of sexual signal evolution in a macroevolutionary framework. These can then be used to gain a greater understanding of the evolution of sexual dimorphism in the Phasianidae and other groups of organisms, potentially revealing any unifying themes in the evolution of sexual signals over relatively large time scales.

\section{Acknowledgments}

This paper was greatly improved by helpful suggestions from an anonymous reviewer and Rob Kulathinal. This research was facilitated by funding from the National Science Foundation (DEB-0228682) to R. T. Kimball, E. L. Braun, and D. W. Steadman.

\section{References}

[1] M. Andersson, Sexual Selection, Princeton University Press, Princeton, NJ, USA, 1994.

[2] U. Candolin, "The use of multiple cues in mate choice," Biological Reviews of the Cambridge Philosophical Society, vol. 78, no. 4, pp. 575-595, 2003.

[3] J. Bro-Jørgensen, "Dynamics of multiple signalling systems: animal communication in a world in flux," Trends in Ecology and Evolution, vol. 25, no. 5, pp. 292-300, 2010.

[4] A. P. Møller and A. Pomiankowski, "Why have birds got multiple sexual ornaments?" Behavioral Ecology and Sociobiology, vol. 32, no. 3, pp. 167-176, 1993.

[5] R. A. Johnstone, "The tactics of mutual mate choice and competitive search," Behavioral Ecology and Sociobiology, vol. 40, no. 1, pp. 51-59, 1997.

[6] T. Guilford and M. S. Dawkins, "Receiver psychology and the design of animal signals," Trends in Neurosciences, vol. 16, no. 11, pp. 430-436, 1993.

[7] T. Guilford and M. S. Dawkins, "Receiver psychology and the evolution of animal signals," Animal Behaviour, vol. 42, no. 1, pp. 1-14, 1991.

[8] C. Rowe, "Receiver psychology and the evolution of multicomponent signals," Animal Behaviour, vol. 58, no. 5, pp. 921931, 1999.

[9] H. Kokko, M. D. Jennions, and A. Houde, "Evolution of frequency-dependent mate choice: keeping up with fashion trends," Proceedings of the Royal Society B, vol. 274, no. 1615, pp. 1317-1324, 2007.

[10] B. Holland and W. R. Rice, "Perspective: chase-away sexual selection: antagonistic seduction versus resistance," Evolution, vol. 52 , no. 1 , pp. $1-7,1998$.

[11] G. S. Van Doorn and F. J. Weissing, "Sexual conflict and the evolution of female preferences for indicators of male quality," American Naturalist, vol. 168, no. 6, pp. 742-757, 2006. 
[12] A. Pomiankowski and Y. Iwasa, "Evolution of multiple sexual preferences by Fisher's runaway process of sexual selection," Proceedings of the Royal Society B, vol. 253, no. 1337, pp. 173181, 1993.

[13] R. O. Prum, "Phylogenetic tests of alternative intersexual selection mechanisms: Trait macroevolution in a polygynous clade (Aves: Pipridae)," American Naturalist, vol. 149, no. 4, pp. 668-692, 1997.

[14] A. Berglund, A. Bisazza, and A. Pilastro, "Armaments and ornaments: an evolutionary explanation of traits of dual utility," Biological Journal of the Linnean Society, vol. 58, no. 4, pp. 385-399, 1996.

[15] R. O. Vasconcelos, J. M. Simões, V. C. Almada, P. J. Fonseca, and M. C. P. Amorim, "Vocal behavior during territorial intrusions in the lusitanian toadfish: boatwhistles also function as territorial 'keep-out' signals," Ethology, vol. 116, no. 2, pp. 155-165, 2010.

[16] A. S. Chaine and B. E. Lyon, "Intrasexual selection on multiple plumage ornaments in the lark bunting," Animal Behaviour, vol. 76, no. 3, pp. 657-667, 2008.

[17] C. Rowe, "Sound improves visual discrimination learning in avian predators," Proceedings of the Royal Society B, vol. 269, no. 1498, pp. 1353-1357, 2002.

[18] J. A. Pfaff, L. Zanette, S. A. MacDougall-Shackleton, and E. A. MacDougall-Shackleton, "Song repertoire size varies with HVC volume and is indicative of male quality in song sparrows (Melospiza melodia)," Proceedings of the Royal Society B, vol. 274, no. 1621, pp. 2035-2040, 2007.

[19] J. T. Thompson, A. N. Bissell, and E. P. Martins, "Inhibitory interactions between multimodal behavioural responses may influence the evolution of complex signals," Animal Behaviour, vol. 76, no. 1, pp. 113-121, 2008.

[20] J. Hoglund, "Size and plumage dimorphism in lek-breeding birds: a comparative analysis," American Naturalist, vol. 134, no. 1, pp. 72-87, 1989.

[21] International Chicken Genome Sequencing Consortium, "Sequence and comparative analysis of the chicken genome provide unique perspectives on vertebrate evolution," Nature, vol. 432, no. 7018, pp. 695-716, 2004.

[22] R. A. Dalloul, J. A. Long, A. V. Zimin et al., "Multi-platform next-generation sequencing of the domestic turkey (Meleagris gallopavo): genome assembly and analysis," PLoS Biology, vol. 8, no. 9, Article ID e1000475, 2010.

[23] S. Madge and P. McGowan, Pheasants, Partridges and Grouse, Princeton University Press, Princeton, NJ, USA, 2002.

[24] G. W. H. Davison, "Avian spurs," Journal of Zoology, vol. 206, pp. 353-366, 1985.

[25] R. T. Kimball and E. L. Braun, "A multigene phylogeny of Galliformes supports a single origin of erectile ability in nonfeathered facial traits," Journal of Avian Biology, vol. 39, no. 4, pp. 438-445, 2008.

[26] R. T. Kimball, E. L. Braun, J. D. Ligon, E. Randi, and V. Lucchini, "Using molecular phylogenetics to interpret evolutionary changes in morphology and behavior in the Phasianidae," Acta Zoologica Sinica, vol. 52, supplement, pp. 362-365, 2006.

[27] J. D. Ligon, R. Kimball, and M. Merola-Zwartjes, "Mate choice by female red junglefowl: the issues of multiple ornaments and fluctuating asymmetry," Animal Behaviour, vol. 55, no. 1, pp. 41-50, 1998.

[28] J. D. Ligon, R. Thornhill, M. Zuk, and K. Johnson, "Male-male competition, ornamentation and the role of testosterone in sexual selection in red jungle fowl," Animal Behaviour, vol. 40, no. 2, pp. 367-373, 1990.
[29] T. S. Johnsen, M. Zuk, and E. A. Fessler, "Social dominance, male behaviour and mating in mixed-sex flocks of red jungle fowl," Behaviour, vol. 138, no. 1, pp. 1-18, 2001.

[30] M. Petrie, H. Tim, and S. Carolyn, "Peahens prefer peacocks with elaborate trains," Animal Behaviour, vol. 41, no. 2, pp. 323-331, 1991.

[31] A. Loyau, M. S. Jalme, and G. Sorci, "Intra- and intersexual selection for multiple traits in the peacock (Pavo cristatus)," Ethology, vol. 111, no. 9, pp. 810-820, 2005.

[32] M. Takahashi, H. Arita, M. Hiraiwa-Hasegawa, and T. Hasegawa, "Peahens do not prefer peacocks with more elaborate trains," Animal Behaviour, vol. 75, no. 4, pp. 12091219, 2008.

[33] R. Buchholz, "Male dominance and variation in fleshy head ornamentation in wild turkeys," Journal of Avian Biology, vol. 28, no. 3, pp. 223-230, 1997.

[34] R. Buchholz, "Female choice, parasite load and male ornamentation in wild turkeys," Animal Behaviour, vol. 50, no. 4, pp. 929-943, 1995.

[35] C. Mateos, "Sexual selection in the ring-necked pheasant: a review," Ethology Ecology and Evolution, vol. 10, no. 4, pp. 313332, 1998.

[36] L. M. Brodsky, "Ornament size influences mating success in male rock ptarmigan," Animal Behaviour, vol. 36, no. 3, pp. 662-667, 1988.

[37] K. Holder and R. Montgomeri, "Context and consequences of comb displays by male rock ptarmigan," Animal Behaviour, vol. 45, no. 3, pp. 457-470, 1993.

[38] P. T. Rintamäki, J. Höglund, E. Karvonen et al., "Combs and sexual selection in black grouse (Tetrao tetrix)," Behavioral Ecology, vol. 11, no. 5, pp. 465-471, 2000.

[39] C. G. Sibley and J. E. Ahlquist, Phylogeny and Classification of Birds: A Study in Molecular Evolution, Yale University Press, New Haven, Conn, USA, 1990.

[40] P. R. Lowe, "Some preliminary notes on the anatomy and systematic position of Afropavo congensis Chapin," in Proceedings of the 9th International Ornithological Congress, J. Delacour, Ed., pp. 219-230, Rouen, France, 1938.

[41] J. Delacour, The Pheasants of the World, Saiga, Surrey, UK, 1977.

[42] R. T. Kimball, E. L. Braun, P. W. Zwartjes, T. M. Crowe, and J. D. Ligon, "A molecular phylogeny of the pheasants and partridges suggests that these lineages are not monophyletic," Molecular Phylogenetics and Evolution, vol. 11, no. 1, pp. 3854, 1999.

[43] T. M. Crowe, R. C. K. Bowie, P. Bloomer et al., "Phylogenetics, biogeography and classification of, and character evolution in, gamebirds (Aves: Galliformes): effects of character exclusion, data partitioning and missing data," Cladistics, vol. 22, no. 6, pp. 495-532, 2006.

[44] M. H. Armstrong, E. L. Braun, and R. T. Kimball, "Phylogenetic utility of avian ovomucoid intron G: a comparison of nuclear and mitochondrial phylogenies in Galliformes," Auk, vol. 118, no. 3, pp. 799-804, 2001.

[45] E. J. Smith, L. Shi, and Z. Tu, "Gallus gallus aggrecan gene-based phylogenetic analysis of selected avian taxonomic groups," Genetica, vol. 124, no. 1, pp. 23-32, 2005.

[46] W. A. Cox, R. T. Kimball, and E. L. Braun, "Phylogenetic position of the New World quail (Odontophoridae): eight nuclear loci and three mitochondrial regions contradict morphology and the Sibley-Ahlquist tapestry," Auk, vol. 124, no. 1, pp. 71-84, 2007. 
[47] V. B. Kaiser, M. van Tuinen, and H. Ellegren, "Insertion events of CR1 retrotransposable elements elucidate the phylogenetic branching order in galliform birds," Molecular Biology and Evolution, vol. 24, no. 1, pp. 338-347, 2007.

[48] J. O. Kriegs, A. Matzke, G. Churakov et al., "Waves of genomic hitchhikers shed light on the evolution of gamebirds (Aves: Galliformes)," BMC Evolutionary Biology, vol. 7, article no. 190, 2007.

[49] Y. Y. Shen, L. Liang, Y. B. Sun et al., "A mitogenomic perspective on the ancient, rapid radiation in the Galliformes with an emphasis on the Phasianidae," BMC Evolutionary Biology, vol. 10, no. 1, article no. 132, 2010.

[50] T. Lislevand, J. Figuerola, and T. Székely, "Evolution of sexual size dimorphism in grouse and allies (Aves: Phasianidae) in relation to mating competition, fecundity demands and resource division," Journal of Evolutionary Biology, vol. 22, no. 9, pp. 1895-1905, 2009.

[51] H. N. Bryant, "The cladistic of matrix representation with parsimony analysis," in Phylogenetic Supertrees. Combining Information to Reveal the Tree of Life Computational Biology, O. R. P. Binnida-Emonds, Ed., vol. 3, pp. 353-368, Kluwer Academic Publishers, Dordrecth, The Netherlands, 2004.

[52] J. Gatesy and M. S. Springer, "A critique of matrix representation with parsimony supertrees," in Phylogenetic Supertrees. Combining Information to Reveal the Tree of Life Computational Biology, O. R. P. Binnida-Emonds, Ed., vol. 3, pp. 369-388, Kluwer Academic Publishers, Dordrecth, The Netherlands, 2004.

[53] C. Camacho, G. Coulouris, V. Avagyan et al., "BLAST+: architecture and applications," BMC Bioinformatics, vol. 10, article no. 421, 2009.

[54] E. Randi, V. Lucchini, A. Hennache, R. T. Kimball, E. L. Braun, and J. D. Ligon, "Evolution of the mitochondrial DNA control region and cytochrome $b$ genes and the inference of phylogenetic relationships in the avian genus Lophura (Galliformes)," Molecular Phylogenetics and Evolution, vol. 19, no. 2, pp. 187-201, 2001.

[55] A. Stamatakis, "RAxML-VI-HPC: maximum likelihood-based phylogenetic analyses with thousands of taxa and mixed models," Bioinformatics, vol. 22, no. 21, pp. 2688-2690, 2006.

[56] A. Stamatakis, "Phylogenetic models of rate heterogeneity: a high performance computing perspective," in Proceedings of 20th IEEE/ACM International Parallel and Distributed Processing Symposium (IPDPS '06), Rhodos, Greece, April 2006.

[57] J. P. Huelsenbeck and F. Ronquist, "MRBAYES: Bayesian inference of phylogenetic trees," Bioinformatics, vol. 17, no. 8, pp. 754-755, 2001.

[58] F. Ronquist and J. P. Huelsenbeck, "MrBayes 3: Bayesian phylogenetic inference under mixed models," Bioinformatics, vol. 19, no. 12, pp. 1572-1574, 2003.

[59] P. A. Johnsgard, Grouse and Quails of North America, University of Nebraska Press, Lincoln, Neb, USA, 1973.

[60] P. A. Johnsgard, The Quails, Partridges, and Francolins of the World, Oxford University Press, Oxford, UK, 1988.

[61] P. A. Johnsgard, The Pheasants of the World, Oxford University Press, Oxford, UK, 1986.

[62] M. Pagel, A. Meade, and D. Barker, "Bayesian estimation of ancestral character states on phylogenies," Systematic Biology, vol. 53, no. 5, pp. 673-684, 2004.

[63] "Mesquite: A modular system for evolutionary analysis Version Version 2.72," http://mesquiteproject.org.
[64] S. J. Hackett, R. T. Kimball, S. Reddy et al., "A phylogenomic study of birds reveals their evolutionary history," Science, vol. 320, no. 5884, pp. 1763-1768, 2008.

[65] M. Pagel, "Detecting correlated evolution on phylogenies: a general method for the comparative analysis of discrete characters," Proceedings of the Royal Society B, vol. 255, no. 1342, pp. 37-45, 1994.

[66] R. T. Kimball, E. L. Braun, D. J. Ligon, V. Lucchini, and E. Randi, "A molecular phylogeny of the peacock-pheasants (Galliformes: Polyplectron spp.) indicates loss and reduction of ornamental traits and display behaviours," Biological Journal of the Linnean Society, vol. 73, no. 2, pp. 187-198, 2001.

[67] A. J. Bonilla, E. L. Braun, and R. T. Kimball, "Comparative molecular evolution and phylogenetic utility of 3/-UTRs and introns in Galliformes," Molecular Phylogenetics and Evolution, vol. 56, no. 2, pp. 536-542, 2010.

[68] N. Kolm, R. W. Stein, A. Ø. Mooers, J. J. Verspoor, and E. J. A. Cunningham, "Can sexual selection drive female life histories? A comparative study on galliform birds," Journal of Evolutionary Biology, vol. 20, no. 2, pp. 627-638, 2007.

[69] S. H. Eo, O. R. P. Bininda-Emonds, and J. P. Carroll, "A phylogenetic supertree of the fowls (Galloanserae, Aves)," Zoologica Scripta, vol. 38, no. 5, pp. 465-481, 2009.

[70] J. J. Wiens, "Widespread loss of sexually selected traits: How the peacock lost its spots," Trends in Ecology and Evolution, vol. 16, no. 9, pp. 517-523, 2001.

[71] R. Buchholz, Adaptive Functions of Fleshy Ornamentation in Wild Turkeys and Related Birds, Dept. of Zoology, University of Florida, Gainesville, Fla, USA, 1994.

[72] R. S. Wilson, C. H. Condon, G. David, S. FitzGibbon, A. C. Niehaus, and K. Pratt, "Females prefer athletes, males fear the disadvantaged: different signals used in female choice and male competition have varied consequences," Proceedings of the Royal Society B, vol. 277, no. 1689, pp. 1923-1928, 2010. 

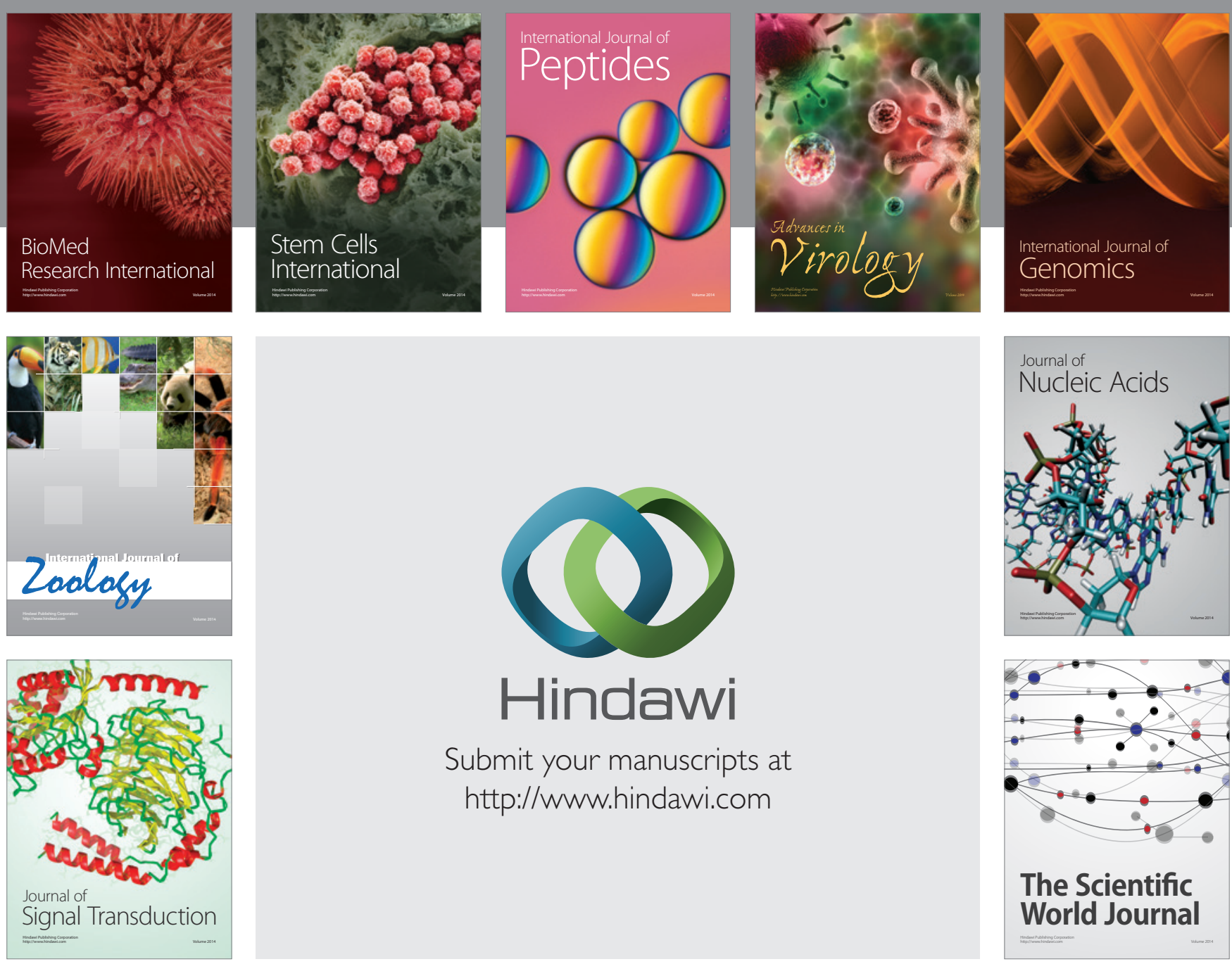

Submit your manuscripts at

http://www.hindawi.com
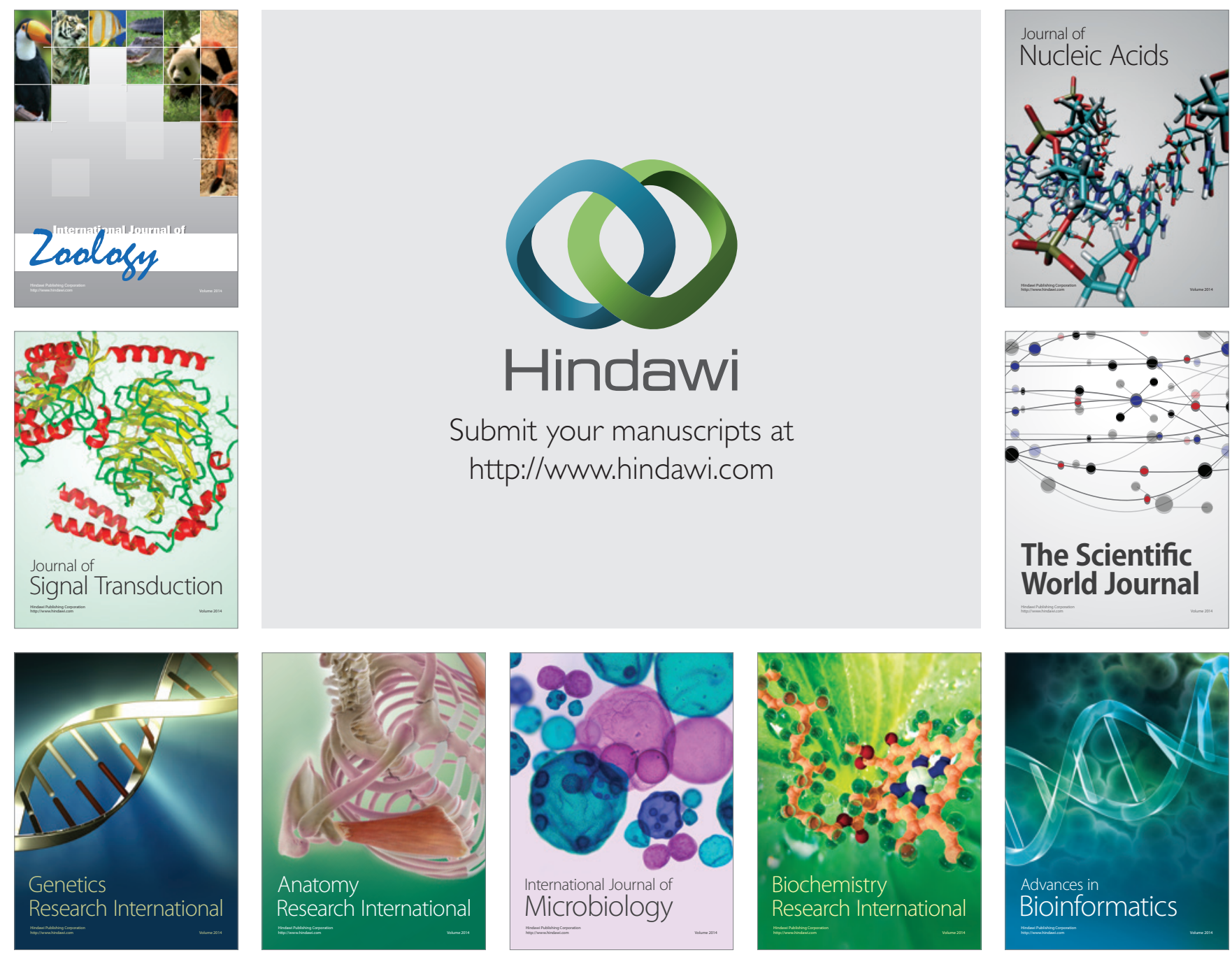

The Scientific World Journal
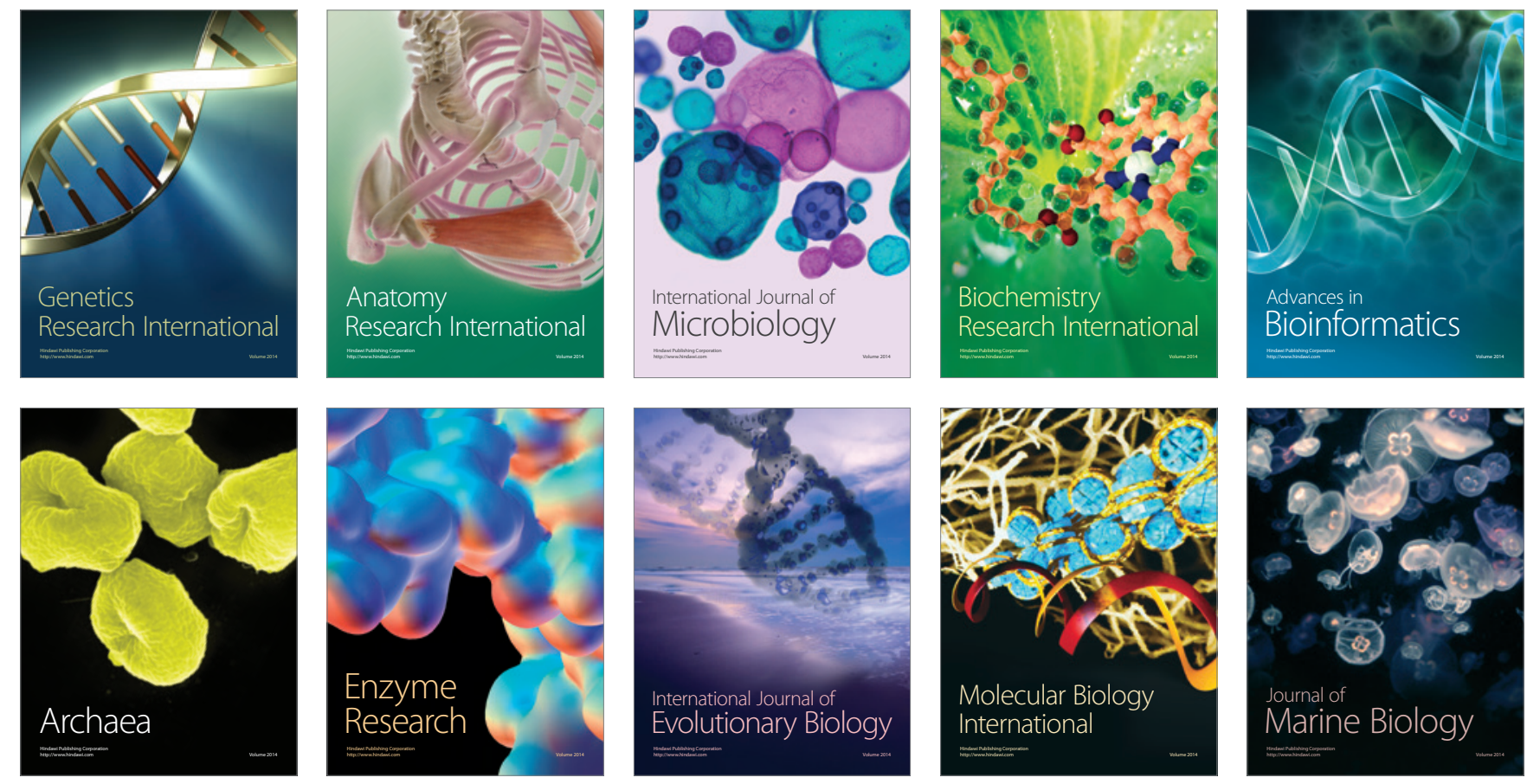